\title{
Sunspot cycle prediction using multivariate regression and binary mixture of Laplace distribution model
}

\author{
A SAbarinath* and A K Anilkumar \\ Applied Mathematics Division, Vikram Sarabhai Space Centre, ISRO, Thiruvananthapuram 695022, India. \\ ${ }^{*}$ Corresponding author. e-mail: a_sabarinath@yahoo.co.in
}

MS received 21 October 2016; revised 28 November 2017; accepted 22 December 2017;

published online 2 August 2018

Prediction of sunspot cycle is a vital activity in space mission planning and various engineering decision making. In the present study, the sunspot cycle prediction has been carried out by a hybrid model which employs multivariate regression technique and the binary mixture of Laplace distribution (BMLD) function. The Expectation Maximization (EM) algorithm is being applied to the multivariate regression analysis to obtain a robust prediction of the sunspot cycle. Sunspot cycle 24 has been predicted using this technique. Multivariate regression model has been derived based on the available cycles 1 to 23 . This model could predict cycle 24 as an average of previous cycles. Prediction from this model has been refined to capture the cycle characteristics such as bimodal peak at the high solar activity period by incorporating a predicted peak sunspot number from the BMLD model. This revised prediction has shown more accuracy in forecasting the major discrete features of sunspot cycle like maximum amplitude, the Gnevyshev gap, time duration from peak to peak amplitude, and the epoch of peak amplitude. This refined prediction shows that cycle 24 will be having a peak amplitude of 78 with an uncertainty of \pm 25 . Moreover, the present forecast says that, cycle 24 will be having double peak with a strong second peak compared to the first peak. This hypothesis is found to be true with the realized data of cycle 24 . Further, this techniques have been validated by predicting sunspot cycles 22 and 23. A preliminary level prediction of sunspot cycle 25 also been carried out using the technique presented here. Present study predicts that, cycle 25 also will be a modest cycle like the present cycle 24 , and the peak amplitude may vary in a band of $75-95$.

Keywords. Sunspot number cycles; multivariate regression; Laplace distribution.

\section{Introduction}

Sunspots appear as dark patches on the visible surface of the Sun (Withbroe 1989). Today, it is understood that these spots are generated by very high concentrations of magnetism, which impede the flow of heat from inside the Sun to its surface. The appearance of sunspots are not a permanent phenomenon on the solar surface. The total number of sunspots on the Sun's visible surface varies cyclically in time, with a period of approximately eleven years. This variation influence the Earth's climate (Saxena et al. 2016).

Solar activity is the key factor which drives the space weather. Refined modeling and accurate prediction of the solar activity intensity have been an important activity of space scientists across the globe due to the impact of solar activity on satellite life time as well as on weather (Petrovay 2010). Solar flux causes the upper atmosphere 
density variation and in turn, it affects directly the lifetime of the Earth-orbiting satellites, especially in low Earth orbit. During high solar activity period, orbital decay of the satellites become faster, and this results in the operational life span reduction and hence increase in the cost of satellite operations (King Hele 1987). With this prime reason, the solar activity prediction is an unavoidable activity in spacecraft mission design and planning (Hathaway 2010).

Direct observations of Sun's activity in the form of various indicators are available since the eighteenth century onwards. Among the various proxy indicators of solar activity, sunspot numbers bear an important role. Another most prominent indicator is the F10.7 cm value. This is a modern proxy indicator which measures the solar flux density. Similarly, there are many indicators available in the literature, like total solar irradiance, solar flare, coronal mass ejections, etc. Among all these, sunspot numbers is the most studied indicator, since it is available from 1749 onwards. Between these parameters, one can find a very good correlation. Joshi et al. (2015) gives a detailed study on this aspect. The flare index correlates well with various parameters of the solar activity. It is found that the flare index exhibits a significantly high correlation with sunspot area over sunspot number, which suggests the variations in sunspot area to be more closely linked with the transient energy release in the solar corona. The time evolution of the solar flare index also confirms that dual peaks can occur in solar cycles during the solar maxima. Scientists have proposed several models and prediction techniques for forecasting this time series well in advance (Pesnell 2008).

Generally, methods of forecasting the sunspot numbers can be classified into two different categories. One is the long term prediction, and the second is the short term prediction (Pesnell 2008; Petrovay 2010). In the long term prediction of sunspot numbers, the objective is to forecast the solar activity for the next one entire solar cycle or many cycles ahead. That is, a long term prediction should forecast the major characteristics of a full cycle just prior to the cycle beginning or at least in the initial period of the cycle or features of many number of cycles. The major characteristics to be forecasted include peak amplitude (maximum value of the sunspot number in a cycle), length of the sunspot cycle, ascent phase length, descent phase length, etc.
Long term prediction of solar activity is useful for mission planning of low Earth orbiting satellites, since in the entire operating period of the low Earth orbiting satellite has to function through various phases of a solar cycle (Withbroe 1989).

In short term prediction, the forecast is for a period of maximum one month from the time of prediction. Unlike the long term prediction, here the prediction of actual magnitude is mostly preferred. This prediction is required daily, because every day from the space many space debris risk objects are reentering into the Earth's atmosphere. The reentry object's impact location on the Earth in terms of latitude and longitude and time of the reentry are to be predicted on a continuous basis. Due to the difficulty in estimating the correct ballistic coefficient, a parameter which is closely associated with the solar activity, the reentry prediction become a difficult task. In fact, drag force on a satellite operating in the low Earth orbit is a function of the solar activity (Drecher et al. 1980), and in precise it depends on the F10.7 cm value. All the reentry prediction software use the solar activity intensity as evaluated by the proxy index $\mathrm{F} 10.7 \mathrm{~cm}$ value. In literature (Hathaway 2010), it had been established that the F10.7 $\mathrm{cm}$ value and sunspot numbers $(R)$ are highly correlated and the model relating these two solar activity indicators is given in equation (1).

$\mathrm{F} 10.7=67+0.97 R+17.6(e-0.035 R-1)$.

This shows that, once the sunspot numbers are available the corresponding solar flux value (F10.7 cm value) can be computed using equation (1). Hence, a prediction model for sunspot numbers is very much useful for the prediction of F10.7 cm values. Since sunspot number data is available for the last three centuries, model building and analysis can be carried out for sunspot numbers. In the present study, an attempt is being made to predict the sunspot number cycle in a long term as well as in a short term manner using a combination of two models, one derived from a multivariate regression and another one derived from the binary mixture of Laplace distribution (BMLD) function (Sabarinath and Anilkumar 2008). Before developing this hybrid model, in the subsequent section we review the various techniques already available in the literature. 


\section{Sunspot cycle prediction techniques - a review}

Classification of the sunspot cycle prediction is done primarily in five ways (Pesnell 2008). They are: (1) climatology, (2) precursor, (3) dynamo model, (4) spectral, and (5) neural networks. In climatological method (Wang et al. 2002; Osherovich and Fainberg 2008), forecasts assume that the future of a system can be determined from the statistical properties of the past. A simple prediction of a future cycle can be the average of the previous few cycles. Many times these kinds of prediction do not consider the local variations especially during the evolution of sunspot cycle. Hence, these models can be refined by incorporating information about the local irregularities.

Precursor methods are most useful categories of prediction techniques. In precursor method (Schatten 2005; Javaraiah 2008; Obridko and Shelting 2008), some indicators of the previous cycles are considered and their relationships to the future cycle characteristics are modeled. Using this model, the future sunspot cycle characteristics are predicted. Geomagnetic activity near the minimum is a good precursor for the level of solar activity for the next maximum.

Dynamo models are physics based and recently many promising studies have been published (Choudhuri et al. 2007; Kitiashvili and Kosovichev 2008; Choudhuri 2014).

Spectral methods (Kane 1999; de Mayer 2003; Kane 2007; Hiremath 2008; Kilicik et al. 2009) examine a Fourier analysis of the sunspot number time series for invariant quantities such as frequencies whose amplitudes are conserved or have simple time dependence. Wavelet based and autoregressive forecasts were considered as spectral.

Finally neural network forecasts (Quassim et al. 2007; Parsapoor et al. 2015) are derived from nonlinear, statistical algorithms that determine and model complex relationships between inputs and outputs to find patterns in the data that can be extrapolated.

Dikpati et al. (2006) predicted the strength of solar cycle 24 using a flux-transport dynamo based tool. They constructed a prediction tool for predicting the solar cycle strength by modifying a calibrated flux-transport dynamo model, and made a prediction of the amplitude. Their prediction shows cycle 24 will have a 30 to $50 \%$ higher peak than cycle 23. But later, when cycle reached its peak it is found that, cycle 24 is weak compared with cycle 23. Choudhuri et al. (2007), used the flux-transport dynamo models and predicted the peak sunspot number of cycle 24 as 80 .

Qian et al. (2006) proposed a thermosphere density based prediction of solar cycle 24 . Here the prediction provides only an estimate of the intensity of cycle 24, but not the shape variation of the cycle. Schatten (2005) made a prediction of solar cycle 24 for smoothed sunspot numbers and F10.7 cm values based on a polar field precursor method. Using this method the peak amplitude of the solar cycle 24 is estimated at $124 \pm 30$ in terms of smoothed F10.7 cm radio flux and $80 \pm 30$ in terms of smoothed international or Zurich sunspot number. Duhau (2003) predicted the maximum sunspot number in solar cycle 24 as $87.5 \pm 23.5$. They used a wavelet analysis on geomagnetic index $(a a)$ and the yearly averaged Wolf sunspot numbers since 1844 and found that, a non-linear coupling function between sunspot maxima and aa minima modulations. Hiremath (2006) modeled the solar cycle as a forced and damped harmonic oscillator and from all the 22 cycles (1755-1996), long-term amplitudes, frequencies, phases and decay factor are obtained. Using these physical parameters of the previous 22 solar cycles and by an autoregressive model, they predict the amplitude and period of the future 15 solar cycles. According to their prediction, the period and amplitude of the cycle 24 are $9.34 \mathrm{yr}$ and $110( \pm 11)$. Cliverd et al. (2006) used a model for sunspot number using low-frequency solar oscillations, with periods $22,53,88,106,213$, and $420 \mathrm{yr}$ modulating the 11-year Schwabe cycle, to predict the peak sunspot number of cycle 24 and for future cycles. Their prediction of cycle 24 is significantly smaller than cycle 23 , with peak sunspot numbers as $42 \pm 34$.

Parsapoor et al. (2015) proposed a prediction of solar cycle 24 using a connectionist model of the emotional system. According to this prediction, the amplitude of the first peak is 87.8 , and the second peak is 95.38. Using direct polar field measurements available for solar cycles 20 to 23, Svalgaard et al. (2005) predicted that the approaching solar cycle 24 will have a peak smoothed monthly sunspot number of 75 with an uncertainty of \pm 8 . This made them to claim that cycle 24 will be the smallest cycle in the last 100 yrs. They used the strength of the polar fields during the decay of one cycle and is assumed to be an indicator of peak sunspot activity for the following cycle. Using nonlinear dynamical techniques, Kilicik et al. (2009) made a prediction of sunspot cycle 24 . Their study 
Table 1. Summary of various predictions for the maximum amplitude of sunspot cycle 24.

\begin{tabular}{|c|c|c|}
\hline Class of model & Reference & $\begin{array}{c}\text { Predicted maximum } \\
\text { amplitude }\end{array}$ \\
\hline Climatological & Osherovich and Fainberg (2008) & $77 \pm 15$ \\
\hline Climatological & Wang et al. (2002) & 83.2 to 119.4 \\
\hline Precursor & Obridko and Shelting (2008) & $105 \pm 10$ \\
\hline Precursor & Javaraiah (2008) & $74 \pm 10$ \\
\hline Precursor & Schatten (2005) & $80 \pm 30$ \\
\hline Precursor & Schatten (2005) & $80 \pm 30$ \\
\hline Precursor & Svalgaard et al. (2005) & $70 \pm 2$ \\
\hline Precursor & Kane (2007) & $142 \pm 24$ \\
\hline Dynamo models & Dikpati et al. (2006) & 155 to 180 \\
\hline Dynamo models & Kitiashvili and Kosovichev (2008) & 85 \\
\hline Dynamo models & Choudhuri et al. (2007) & 80 \\
\hline Spectral methods & Hiremath (2008) & $110 \pm 11$ \\
\hline Spectral methods & de Mayer (2003) & $110 \pm 15$ \\
\hline Spectral methods & Kane (1999) & $105 \pm 9$ \\
\hline Spectral methods & Kilicik et al. (2009) & 87.4 \\
\hline Nueral networks & Quassim et al. (2007) & 110 \\
\hline Nueral networks & Parsapoor et al. (2015) & 88 \\
\hline Nueral networks & Cliverd et al. (2006) & $42 \pm 35$ \\
\hline Wavelet analysis & Duhau (2003) & $87.5 \pm 23.5$ \\
\hline Nonlinear methods & Kilicik et al. (2009) & 87 \\
\hline Statistical & Wang et al. (2002) & 83.2 to 119.4 \\
\hline
\end{tabular}

explores the trends in sunspot activity, using new techniques based on non-linear methods and made a decadal scale prediction of the solar cycle 24 . First, Hurst exponent analysis is used to investigate the autocorrelation structure of the putative dynamics, then the Sugihara-May algorithm is used to predict the ascent phase time and the maximum intensity of the sunspot cycle 24 . According to this method, the prediction of peak amplitude of cycle 24 is 87 and cycle 24 reach to its peak amplitude in December 2012.

Wang et al. (2002) predicted that the maximum amplitude of solar cycle 24 will vary in a band from 83.2 to 119.4. They used statistical characteristic of sunspot cycles in developing the prediction techniques. For this, the solar cycles are divided into two groups, a high rising velocity group and a low rising velocity group, depending on the rising velocity in the ascending phase for a given duration of the ascending phase. Then the amplitude of solar cycle 24 is predicted using the formula derived in the study. A preliminary estimate of the size of a future solar cycle based on Ohl's precursor method was proposed by Kane (2007). Using this, they predicted cycles 22 and 23. Dabas et al. (2008) proposed a precursor method, which predicts the maximum amplitude of cycle 24 as 124 with and uncertainty of \pm 23 . Further, they predicted that the peak of the cycle 24 will be occurring about 45 months after the occurrence of its minimum amplitude with an uncertainty of \pm 4 months. Table 1 gives a summary of the various predictions discussed here.

Having reviewed various modeling and prediction methods, it is found that, many of these models lack the ability to predict a complete sunspot cycle, which can be directly used in applications like life time estimation of space objects. Many of the models described above predicts a future cycle characteristic parameters (for example, peak amplitude, length, etc.) very efficiently. But they cannot forecast an entire complete cycle. In the real world applications, like satellite mission planning, satellite life time estimation process, etc., forecasted complete sunspot cycles are required. The present study can predict a complete sunspot cycle. Here, the sunspot cycle prediction has been carried out by a hybrid model, which is based on the multivariate regression technique and the BMLD function as a shape model of the sunspot cycle. Using multivariate regression technique, prediction of a complete cycle has been obtained in maximum likelihood sense. The Expectation Maximization (EM) algorithm (Little and Rubin 1987) is applied to the multivariate regression model to have a probabilistically more accurate prediction 
of the sunspot cycle. The BMLD function predicts the peak value of the next cycle accurately. So we could predict a future cycle end to end using this method very efficiently. With this hybridized model the sunspot cycles 24 and 25 have been predicted. In fact, two predictions for the full sunspot cycle 25 are provided based on the initial characteristics of cycle 25 .

\section{Sunspot number data, transformed data and analysis}

In the present study, the predictions of sunspot cycles are carried out with the monthly averaged sunspot number values. Since the maximum amplitudes of sunspot cycles are characterized by the maximum monthly smoothed sunspot number obtained by smoothing of monthly sunspot numbers with a 13-month filter, we carried out this analysis with the smoothed monthly averaged sunspot number data provided by NOAA (National Oceanic and Atmospheric Administration). The smoothed sunspot data were available from http://www.sidc.be/silso/versionarchive at the Royal Observatory, Belgium. It may be noted that, the scientific community recently recalibrated the entire historical sunspot number record and that SILSO (Sunspot Index and Long-term Solar Observations) maintains this new record as well as the original version of sunspot numbers.

Table 2 gives a detailed description of the major properties of all the past 23 sunspot cycles in terms of the cycle length, peak amplitude, duration of ascent phase and descent phase, cycle start time, peaking time, etc. It may be seen that the length of the cycles are not exactly $11 \mathrm{yr}$, but on average it is about $11.05 \mathrm{yr}$. Here for the purpose of analysis, we assume each cycle is having length of 132 months ( $\approx 11$ yr). For doing this, a normalization of sunspot cycle data into 132 month is carried out. This enables the computation involved easier. Hence, after the normalization, each of the cycle consist of 132 points, which correspond to 132 months in the 11-yr period. From actual values to the normalized values, the difference in magnitude of sunspot number are not significant. But, the time of occurrence of peak amplitude, duration of rising time and falling time will change due to the length of the cycle is being contracted or elongated due to the normalization. Mathematical details of the process of normalization is given here.

Let an arbitrary sunspot cycle data be represented as $S=\left\{\left(t_{1}, S_{1}\right),\left(t_{2}, S_{2}\right), \ldots,\left(t_{N}, S_{N}\right)\right\}$, where $t_{N}$ is the length of the cycle and $S_{i}, i=1$, $2, \ldots, N$ be the magnitude of the sunspot numbers on each month. Then transform $S$ into $S^{\prime}=$ $\left\{\left(\frac{t_{1} * 132}{t_{N}}, S_{1}\right),\left(\frac{t_{2} * 132}{t_{N}}, S_{2}\right), \ldots,\left(\frac{t_{N} * 132}{t_{N}}, S_{N}\right)\right\}$. Further to make the length of the set $S^{\prime}$ into 132 , interpolate $S^{\prime}$ at $1,2, \ldots, 132$. Then, use this revised (after interpolation) set as the normalized set. This procedure has been carried out for all the 23 sunspot cycles and the major characteristic parameter changes are listed in table 3 . So, once a prediction with the normalized data have been obtained, a renormalizing factor $132 / t_{N}$ has to be multiplied to the time parameter. In the present study, for the next cycle $t_{N}$ is computed as an average of the previous cycles.

Figure 1 shows the normalized sunspot cycle for all the cycles from 1 to 23, along with the averaged cycle and it can be seen from this figure that, the peak amplitude of smoothed sunspot number cycles vary from 50 to 200 . Table 3 gives the difference in the peak sunspot numbers due to normalization. It can be seen that there is no significant change in the magnitude of the peak amplitude due to normalization to 132 points. The change in the other parameters like rise time, fall time are also given in table 3.

In the present study, the prediction technique is developed in two phases. In the first phase we predict the cycle 24 based on a multivariate regression imputation technique (Little and Rubin 1987) and in the second phase we refine this prediction more realistic one by incorporating a prediction based on the model derived from BMLD function. In the next section, the detailed mathematical modeling aspects and algorithm is described.

\section{Methodology and algorithm}

The methodology used here is a computational procedure based on the theory of Expectation Maximization (EM) algorithm (Little and Rubin 1987). The EM algorithm is an iterative algorithm for maximum likelihood estimation in incomplete data problems. It is found to be an efficient method for handling the missing data (Little and Rubin 1987).

In the present study, it is assumed that sunspot numbers to be forecasted (i.e., cycle 24) are missing in the data matrix consisting of known sunspot numbers. Considering the initial two years of data in cycle 24 as available, the remaining sunspot numbers of the cycle can be predicted using 
Table 2. Minima and maxima of sunspot number cycles.

\begin{tabular}{|c|c|c|c|c|c|c|c|}
\hline $\begin{array}{l}\text { Sunspot } \\
\text { cycle } \\
\text { no. }\end{array}$ & $\begin{array}{c}\text { Year of } \\
\text { min }\end{array}$ & $\begin{array}{c}\text { Smallest } \\
\text { smoothed } \\
\text { monthly mean }\end{array}$ & $\begin{array}{c}\text { Year of } \\
\max \end{array}$ & $\begin{array}{c}\text { Largest } \\
\text { smoothed } \\
\text { monthly mean }\end{array}$ & $\begin{array}{l}\text { Rise to } \\
\max \\
(y r s)\end{array}$ & $\begin{array}{c}\text { Fall to } \\
\text { min } \\
(y r s)\end{array}$ & $\begin{array}{l}\text { Cycle } \\
\text { length } \\
(y r s)\end{array}$ \\
\hline 1 & 1755.2 & 8.4 & 1761.5 & 86.5 & 6.3 & 5.0 & 11.3 \\
\hline 2 & 1766.5 & 11.2 & 1769.7 & 115.8 & 3.2 & 5.8 & 9.0 \\
\hline 3 & 1775.5 & 7.2 & 1778.4 & 158.5 & 2.9 & 6.3 & 9.2 \\
\hline 4 & 1784.7 & 9.5 & 1788.1 & 141.2 & 3.4 & 10.2 & 13.6 \\
\hline 5 & 1798.3 & 3.2 & 1805.2 & 49.2 & 6.9 & 5.4 & 12.3 \\
\hline 6 & 1810.6 & 0.0 & 1816.4 & 48.7 & 5.8 & 6.9 & 12.7 \\
\hline 7 & 1823.3 & 0.1 & 1829.9 & 71.7 & 6.6 & 4.0 & 10.6 \\
\hline 8 & 1833.9 & 7.3 & 1837.2 & 146.9 & 3.3 & 6.3 & 9.6 \\
\hline 9 & 1843.5 & 10.5 & 1848.1 & 131.6 & 4.6 & 7.9 & 12.5 \\
\hline 10 & 1856.0 & 3.2 & 1860.1 & 97.9 & 4.1 & 7.1 & 11.2 \\
\hline 11 & 1867.2 & 5.2 & 1870.6 & 140.5 & 3.4 & 8.3 & 11.7 \\
\hline 12 & 1878.9 & 2.2 & 1883.9 & 74.6 & 5.0 & 5.7 & 10.7 \\
\hline 13 & 1889.6 & 5.0 & 1894.1 & 87.9 & 4.5 & 7.6 & 12.1 \\
\hline 14 & 1901.7 & 2.6 & 1907.0 & 64.2 & 5.3 & 6.6 & 11.9 \\
\hline 15 & 1913.6 & 1.5 & 1917.6 & 105.4 & 4.0 & 6.0 & 10.0 \\
\hline 16 & 1923.6 & 5.6 & 1928.4 & 78.1 & 4.8 & 5.4 & 10.2 \\
\hline 17 & 1933.8 & 3.4 & 1937.4 & 119.2 & 3.6 & 6.8 & 10.4 \\
\hline 18 & 1944.2 & 7.7 & 1947.5 & 151.8 & 3.3 & 6.8 & 10.1 \\
\hline 19 & 1954.3 & 3.4 & 1957.9 & 201.3 & 3.6 & 7.0 & 10.6 \\
\hline 20 & 1964.9 & 9.6 & 1968.9 & 110.6 & 4.0 & 7.6 & 11.6 \\
\hline 21 & 1976.5 & 12.2 & 1979.9 & 164.5 & 3.4 & 6.9 & 10.3 \\
\hline 22 & 1986.8 & 12.3 & 1989.6 & 158.5 & 2.8 & 6.8 & 9.7 \\
\hline 23 & 1996.4 & 8.0 & 2000.3 & 120.8 & 4.0 & 8.9 & 12.9 \\
\hline
\end{tabular}

this procedure. This method have been tested by considering sunspot cycles 22 and 23 as a test case and the entire sunspot cycle 24 have been predicted. Before dwelling into the computational details the theoretical background is being reviewed briefly here.

\subsection{Expectation maximization algorithm}

The EM algorithm is a procedure for maximum likelihood estimation in incomplete data problems. The EM algorithm is working based on the following steps: (1) replacing the missing values by estimated values, (2) estimation of parameters, and (3) iterative estimation of the missing values by assuming the new parameter estimates are correct until the parameters converges. Such methods are collectively called as the EM algorithm. In EM algorithms, the complete data likelihood $l\left(\theta \mid Y_{\mathrm{obs}}, Y_{\mathrm{mis}}\right)=\ln L\left(\theta \mid Y_{\mathrm{obs}}, Y_{\mathrm{mis}}\right)$ is linear in $Y_{\mathrm{mis}}$. Where, $L$ is the likelihood function, $Y_{\text {mis }}$ is the missing part of $Y$, and $Y_{\text {obs }}$ is the observed part of $Y$. $\theta$ represents the parameter to be maximized in the maximum likelihood sense. More generally, missing sufficient statistics of individual observations are needed to be estimated or the log likelihood $l(\theta \mid Y)$ itself needs to be estimated at each iteration of the algorithm.

Each iteration of the EM algorithm consists of an E step (expectation step) and an M step (maximization step). An important advantage of the algorithm is that, it converges reliably, in the sense that under the general conditions, each iteration increases the likelihood $l\left(\theta \mid Y_{\text {obs }}\right)$, and if $l\left(\theta \mid Y_{\text {obs }}\right)$ is bounded, the sequence $l\left(\theta^{(t)} \mid Y_{\text {obs }}\right)$ converges to a stationary value of $l\left(\theta \mid Y_{\text {obs }}\right)$ (Little and Rubin 1987).

As described in the above, the EM algorithm has two steps to perform, viz., the E step and the M step. The M-step performs for maximum likelihood estimation of the unknown parameter $\theta$ just as if there were no missing data. The E step finds the conditional expectation of the missing data (values to be predicted) given the observed data and current estimated parameters. The expected values obtained in such a way are then imputed for the missing data.

Specifically, let $\theta^{(t)}$ be the current estimate of the parameter $\theta$. The E step of EM finds the expected $\log$ likelihood if $\theta$ were $\theta^{(t)}$ : 
Table 3. The maximum sunspot number after and before normalization.

\begin{tabular}{|c|c|c|c|c|}
\hline $\begin{array}{l}\text { Cycle } \\
\text { no. }\end{array}$ & $\begin{array}{c}\text { Maximum sunspot } \\
\text { number after } \\
\text { normalization }\end{array}$ & $\begin{array}{c}\text { Maximum sunspot } \\
\text { number before } \\
\text { normalization }\end{array}$ & $\begin{array}{c}\text { Rise time after } \\
\text { normalization }\end{array}$ & $\begin{array}{c}\text { Rise time before } \\
\text { normalization }\end{array}$ \\
\hline 1 & 86.47025 & 86.5 & 6.1 & 6.3 \\
\hline 2 & 115.7006 & 115.8 & 3.9 & 3.2 \\
\hline 3 & 158.2389 & 158.5 & 3.5 & 2.9 \\
\hline 4 & 141.1458 & 141.2 & 2.9 & 3.4 \\
\hline 5 & 49.10968 & 49.2 & 6.4 & 6.9 \\
\hline 6 & 48.57407 & 48.7 & 5.0 & 5.8 \\
\hline 7 & 71.49935 & 71.7 & 6.9 & 6.6 \\
\hline 8 & 146.8994 & 146.9 & 3.9 & 3.3 \\
\hline 9 & 130.9926 & 131.6 & 4.0 & 4.6 \\
\hline 10 & 97.79361 & 97.9 & 4.1 & 4.1 \\
\hline 11 & 140.2919 & 140.5 & 3.3 & 3.4 \\
\hline 12 & 74.53313 & 74.6 & 5.1 & 5.0 \\
\hline 13 & 87.80624 & 87.9 & 4.2 & 4.5 \\
\hline 14 & 64.19149 & 64.2 & 5.2 & 5.3 \\
\hline 15 & 105.3993 & 105.4 & 4.4 & 4.0 \\
\hline 16 & 78.08257 & 78.1 & 5.2 & 4.8 \\
\hline 17 & 119.1394 & 119.2 & 3.8 & 3.6 \\
\hline 18 & 151.7975 & 151.8 & 3.6 & 3.3 \\
\hline 19 & 201.282 & 201.3 & 3.8 & 3.6 \\
\hline 20 & 110.5991 & 110.6 & 3.8 & 4.0 \\
\hline 21 & 164.3105 & 164.5 & 3.7 & 3.4 \\
\hline 22 & 158.4936 & 158.5 & 3.2 & 2.8 \\
\hline 23 & 120.4224 & 120.8 & 3.5 & 4.0 \\
\hline
\end{tabular}

$$
Q\left(\theta \mid \theta^{(t)}\right)=\int l(\theta \mid Y) f\left(Y_{\mathrm{mis}} \mid Y_{\mathrm{obs}}, \theta=\theta^{(t)}\right) d Y_{\mathrm{mis}}
$$

The M step of EM determines $\theta^{(t+1)}$ by maximizing this expected log likelihood

$$
Q\left(\theta^{(t+1)} \mid \theta^{(t)}\right) \geq Q\left(\theta \mid \theta^{(t)}\right), \quad \text { for all } \theta .
$$

The present study of sunspot cycle prediction have been carried out by the EM algorithm as described in Little and Rubin (1987) as maximum likelihood estimation on multivariate normally distributed variables.

\subsection{Algorithm for predicting sunspot cycles}

The prediction of cycle 24 has been obtained by assuming the first 23 sunspot cycles are fully available and cycle 24 is available only for initial two years. Our aim is to predict the remaining portion of cycle 24 , i.e., from the 25 th to 132 nd month. The schematic of this procedure is described in figure 2 .

Let $O$ be the matrix of order $24 \times 132$ formed from normalized sunspot cycles. The first 23 rows

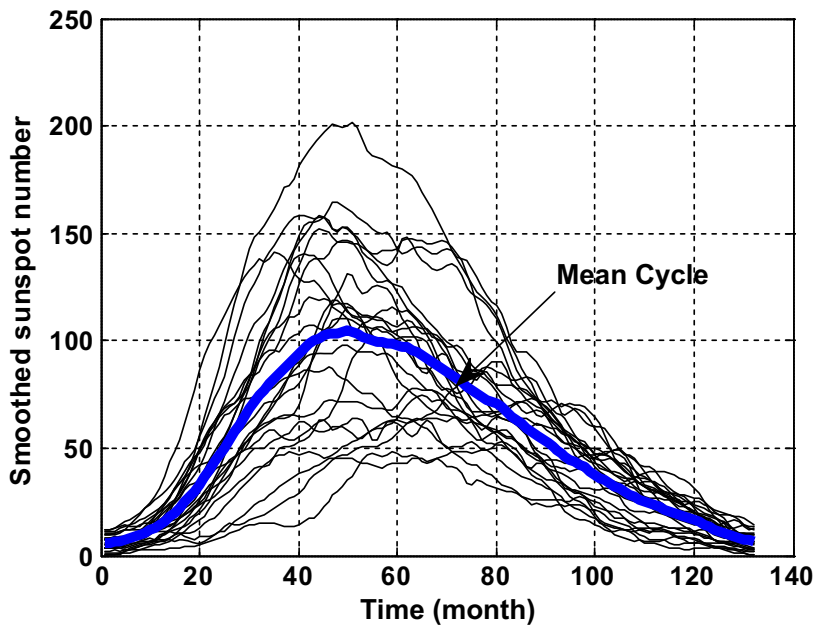

Figure 1. Normalized sunspot cycles 1 to 23 along with the mean sunspot cycle.

of the matrix $O$ consist of the sunspot numbers of cycles 1 to 23 . Row 24 consist of the sunspot cycle corresponds to the 24 th cycle. It may be noted that the first 23 rows are fully observed, since all the cycles 1 to 23 are past cycles. Now the aim is to predict the cycle 24 . To predict the cycle 24 , it requires 


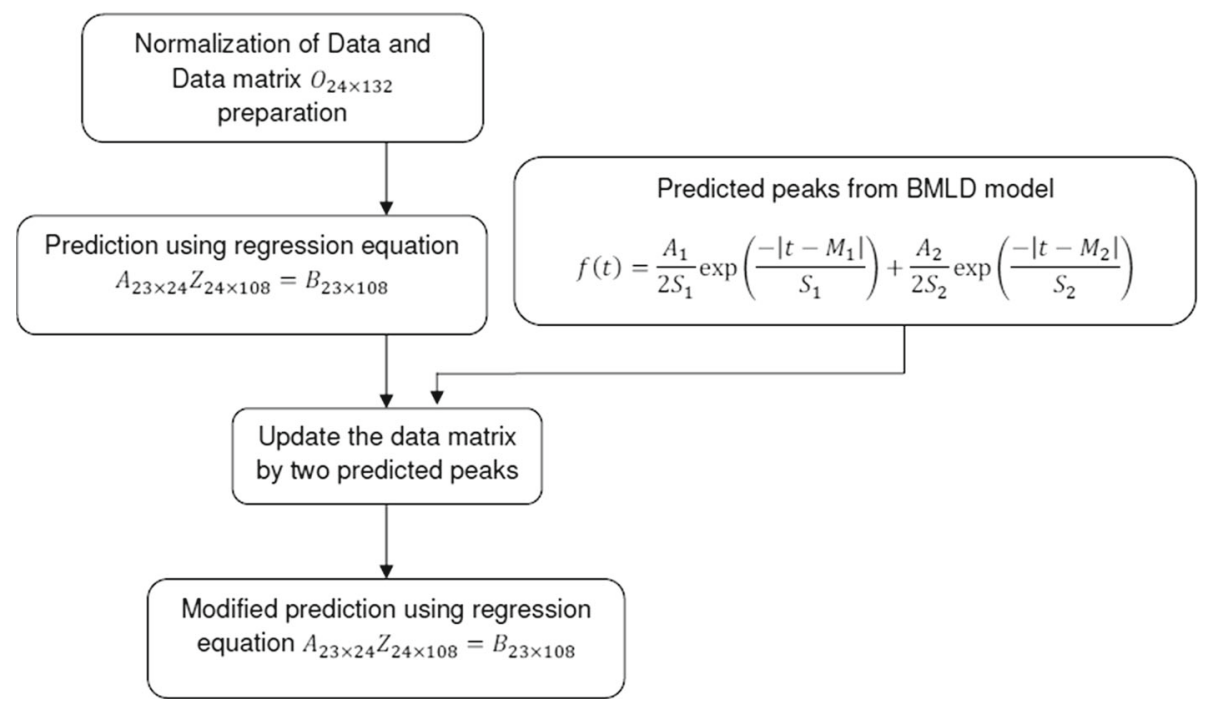

Figure 2. Procedure for the prediction algorithm which uses the peak amplitude predicted by the BMLD model.

some initial realizations of cycle 24 . Otherwise, if the prediction is carried out then the forecasted cycle will be the one which is as close as to the mean prediction as plotted in figure 1 . If we have a few values of initial portion of cycle 24 , then the prediction would be more realistic. So assume that initial two years of data are available. But, since we assume only the initial 24 months of data for cycle 24 , the 24 th row consist of initial 24 data points and from 25 th to 132 nd points are missing (to be predicted).

Now the objective is to predict the missing value, which is the value in column 25th to 132nd of row 24.

For this, let us put this idea in matrix notation as $O=\left[\begin{array}{l}M \\ x_{24}\end{array}\right]$

where $M$ is the matrix of order $23 \times 132$, and $x_{24}$ is the 24 th row of $O$, where the order of $O$ is $24 \times 132$.

Now consider the linear system $A Z=B$, where $A$ is a matrix of order $23 \times 24$ consisting of the first 24 columns of the matrix $M$ and $B$ is the matrix of order $23 \times 108$ consisting of the remaining columns of the matrix $M$, i.e., columns 25 th to 132 nd of the matrix $O$.

Now this is a multivariate regression problem. Here $Z$ consists of the regression coefficients matrix of order $24 \times 108$. Either by the generalized inversion of matrix or through singular value decomposition (SVD), we can compute the solution of $A Z=B$. Let $Z^{\prime}$ be a solution.

Next, consider the augmented system, $\tilde{A} Z=\tilde{B}$, where $\tilde{A}$ is the matrix of order $24 \times 24$ formed by adding one more row as its 24 th row from the initial

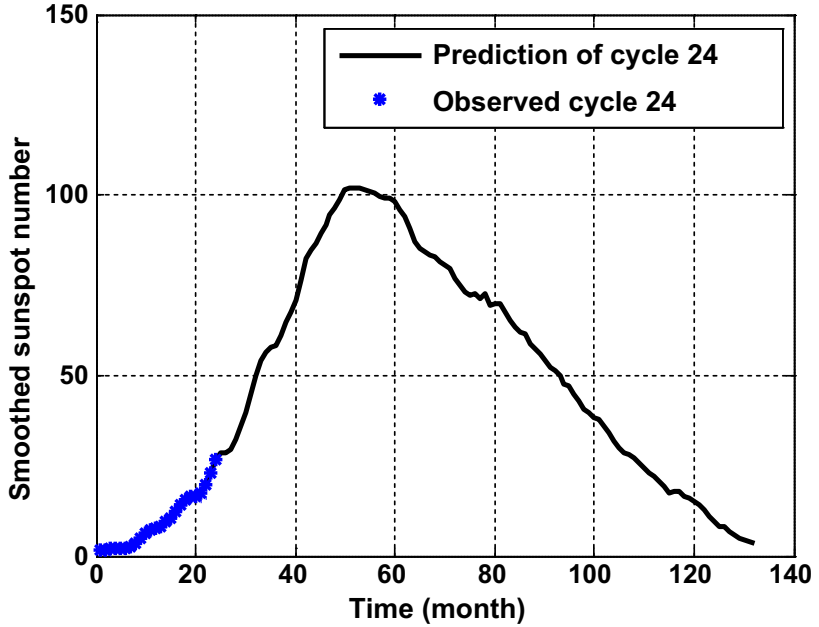

Figure 3. Initial prediction of cycle 24 by utilizing the twoyear data at the beginning of the cycle.

24 data of $x_{24}$, and $\tilde{B}$ is the matrix of order $24 \times 108$ formed by adding the unobserved part of $x_{24}$ (last 108 data) as its 24th row.

Now using $Z^{\prime}$, matrix of order $24 \times 108$, we can easily compute the unobserved part of $\tilde{A} Z=\tilde{B}$. Then treat the system $\tilde{A} Z=\tilde{B}$ is fully observed and recomputed the regression coefficients and again compute the last 107 data of $x_{24}$. Figure 3 shows the prediction of cycle 24 by the above described method.

\section{Prediction improvement}

The deviation of the prediction from the actual observed data can be improved through the following method. Here an improvement on the 
proposed prediction technique is by the predicted peak amplitude from a shape model as proposed in Sabarinath and Anilkumar (2008). Here we consider the prediction from the shape model derived from BMLD function.

Statistical distribution functions gives a class of models for various physical process happening in the world. To model the shape of sunspot number cycles various statistical distribution functions can be selected. In this direction the best suitable functions are (1) Gaussian probability distribution, (2) F-distribution function, (3) $\chi^{2}$-distribution function, and (4) Laplace distribution function. This is mainly due to their shape. But when we select a model for a process, the following criteria should be maintained. They are (1) model should have a minimum number of free parameters, and (2) model should exhibit a high goodness of fit. Goodness of fit for the sunspot cycles models by Hathaway et al. (1994) is measured by the following function

$$
\bar{\chi}=\sqrt{\frac{\left[\sum_{i=1}^{N} \frac{\left(R_{i}-f_{i}\right)^{2}}{s_{i}^{2}}\right]}{N}},
$$

where $R_{i}$ is the monthly averaged sunspot number, $f_{i}$ is the functional fit value, $s_{i}$ is the standard deviation for the monthly averaged sunspot number, $N$ is the number of months in the cycle. The analysis provided in Sabarinath and Anilkumar (2008) shows that the BMLD model gives the best fit with minimum error (within the 0.47 standard deviation of the data points) when comparing with various other models available. By considering these aspects, we propose the BMLD function to model the sunspot number cycle. This is achieved by suitably modifying it in order to fit the sunspot number cycle.

This model is derived from the shape of all previous 23 sunspot cycles. It is evident in almost all sunspot cycles that, especially in the most recent sunspot cycles, multiple peaks are occurring during the peak solar activity period. It is noticed that this model could capture the double peak phenomena, without losing much information around rise and fall time of the sunspot number cycle. The modified BMLD is given in equation (5)

$$
\begin{aligned}
f(t)= & \frac{A_{1}}{2 S_{1}} \exp \left(\frac{-\left|t-M_{1}\right|}{S_{1}}\right) \\
& +\frac{A_{2}}{2 S_{2}} \exp \left(\frac{-\left|t-M_{2}\right|}{S_{2}}\right),
\end{aligned}
$$

where $t$ is the time in units of months from the start of a sunspot number cycle. $M_{1}$ and $M_{2}$ represent the location of the first and second peak during the 11 yr sunspot cycle (one can view these parameters as the beginning and end of the Gnevyshev's gap in a sunspot cycle). The parameters $S_{1}$ and $S_{2}$ are the scale parameters, in the model, represent the curvature in the ascent and descent phase of a sunspot cycle. The remaining two parameters, $A_{1}$ and $A_{2}$ represent the total area under the model curve, which may be termed as the total sunspot content (Sabarinath and Anilkumar 2008). Since the area under the probability distribution function is unity, an area parameters were introduced to fit the model over the actual sunspot numbers. Sabarinath and Anilkumar (2008) give more details on the modified BMLD.

The primary advantage of the BMLD is that it can easily capture the dip due to the Gnevyshev gap at the high solar-activity period. This is a prominent phenomenon in the modern cycles. Gnevyshev (1967) suggested that sunspot cycles have two peaks generated by different physical mechanisms. It has been studied (Kane 2005; Georgieva and Kirov 2011; Joshi et al. 2015) that the double peaks in the past sunspot cycles 12 to 23 are manifestation of the two surges of toroidal field. One generated from the poloidal field from the surface to the poles and another one generated from the poloidal field from the surface to the tachocline. The existence of these two surges of toroidal field is due to the relative magnitudes of the speed of the large-scale solar meridional circulation and the diffusivity in the solar convection zone which are estimated from geomagnetic data. This happens generally from months 35 to 70 of a sunspot number cycle. Due to this multiple maximums, a double peak model can fit the Gnevyshev gap effectively rather than a single peak function. After hybridizing this model along with the multiple regression, an improved prediction of cycle 24 is obtained.

The improvement in the prediction is carried out in the following way. Initially, we classify all the completed sunspot cycles into two groups as fast rising class and slow rising class. If the maximum sunspot number during the initial 24 months is less than 30, we call it as a slow rising cycle. Otherwise, it is called as a fast rising sunspot cycle. The selection of 30 is based on empirical analysis of each individual cycles. For making a prediction improvement, we make two models, viz., one for slow rising class and another one for fast 
rising class. By observing all the 23 completed normalized sunspot cycles, cycles $1,5,6,7,14,16$, and 17 are low raising cycles and the remaining cycles are fast rising cycles. It may be noted that since the 24th month sunspot number value of cycle 17 is almost 30 and hence we consider this cycle also in the slow rising category. The model parameters are derived as the average of all the corresponding parameters of the cycles in the particular class. The derived models for slow rise and fast rise are given in equations (6 and 7), respectively

$$
\begin{aligned}
f(t)= & 48.34 \exp \left(\frac{-|t-45.54|}{13.98}\right) \\
& +71.77 \exp \left(\frac{-|t-74.05|}{27.25}\right) . \\
f(t)= & 103.73 \exp \left(\frac{-|t-38.01|}{13.57}\right) \\
& +94.91 \exp \left(\frac{-|t-65.63|}{27.06}\right) .
\end{aligned}
$$

One typical characteristic of the cycle 24 when compared with the past cycles is its slow ris-

\begin{tabular}{|c|c|c|}
\hline $\begin{array}{l}\text { Sunspot } \\
\text { cycle no. }\end{array}$ & $\begin{array}{l}\text { Normalized } \\
\text { sunspot number } \\
\text { at } 24 \text { th month }\end{array}$ & $\begin{array}{c}\text { Peak } \\
\text { amplitude }\end{array}$ \\
\hline 6 & 8.49 & 48.7 \\
\hline 5 & 10.88 & 49.2 \\
\hline 7 & 11.37 & 71.7 \\
\hline 1 & 19.37 & 86.5 \\
\hline 14 & 26.57 & 64.2 \\
\hline 16 & 29.99 & 78.1 \\
\hline 17 & 30.47 & 119.2 \\
\hline
\end{tabular}
ing nature. If we compare the sunspot value at

Table 4. Sunspot number at 24th month and Peak amplitude for low rising cycles. 24th month for all the previous cycles it can be seen that, cycle 6 is having the minimum value of 8.49 at 24 th month. Hence according to our convention it is a slow rising cycle. Table 4 gives the sunspot number at 24 th month along with the peak sunspot number for all the slow rising cycles.

Hence, we considered these cycles for modeling the double peak phenomena of cycle 24. Table 5 gives the model parameters (location, scale and area parameters) as given in Sabarinath and Anilkumar (2011) of all the slow rising cycles. Further, the shape model for predicting cycle 24 is taken as the average by averaging of model parameters for the cycles $1,5,6,7,14,16$, and 17 . Hence, our shape model for the present study is given in equation (6).

In this model, the parameters are taken as the average of the model parameters of the slow rising cycles. With this model, we could predict cycle 24 as given in figure 4 . It can be seen that for cycle 24, the second peak is more stronger than the first peak. Further, the first peak of cycle 24 will occur at 46th month from the cycle beginning with a magnitude of 72 and the second peak will occur at 74th month from the beginning of the cycle with a magnitude of 78 . To obtain a refined prediction of cycle 24 , consider the value of 72 at 41 st position and the value of 78 at 65 th position. The 41 st and 65 th position is taken as the predicted epoch of cycle peak as given in Sabarinath and Anilkumar (2008). Again through the multiple regression technique, compute the prediction for 24 th to $132 \mathrm{nd}$ month. This improved prediction is plotted in figure 5. Since the 24th cycle is still in progression, at present it is not possible to get the coefficient of renormalization (Length of actual cycle/132), hence a shift in the data point may be seen when we plot the

Table 5. Model parameters of BMLD function for slow rising cycles.

\begin{tabular}{lcrrrrr}
\hline $\begin{array}{l}\text { Sunspot } \\
\text { cycle no. }\end{array}$ & $M_{1}$ & \multicolumn{1}{c}{$S_{1}$} & \multicolumn{1}{c}{$M_{2}$} & \multicolumn{1}{c}{$S_{2}$} & \multicolumn{1}{c}{$A_{1}$} & $A_{2}$ \\
\hline 6 & 54.85 & 8.73 & 74.13 & 30.68 & 134.18 & 2966.22 \\
5 & 49.27 & 15.29 & 79.89 & 23.02 & 1275.34 & 2258.30 \\
7 & 52.01 & 18.84 & 79.44 & 27.20 & 1340.48 & 4108.54 \\
1 & 37.96 & 8.43 & 77.49 & 30.00 & 549.16 & 5543.71 \\
14 & 43.92 & 19.78 & 77.12 & 23.36 & 2710.07 & 2256.10 \\
16 & 35.59 & 15.24 & 61.98 & 25.67 & 1389.97 & 4213.26 \\
17 & 45.20 & 11.58 & 68.30 & 30.84 & 2061.85 & 6033.91 \\
Average & 45.54 & 13.98 & 74.05 & 27.25 & 1351.57 & 3911.43 \\
\hline
\end{tabular}




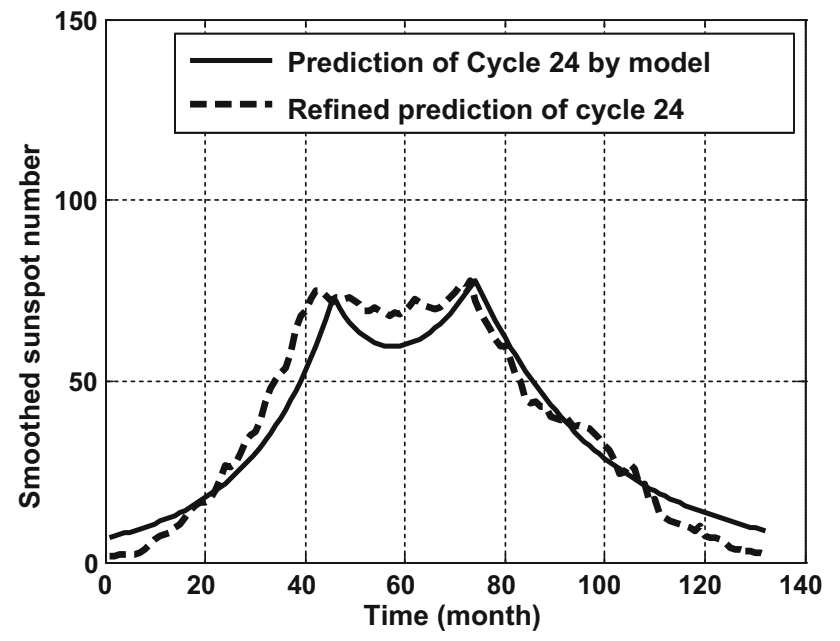

Figure 4. Prediction of sunspot cycle 24 as the averaged BMLD model over the past seven cycles 17 to 23 and refined prediction of cycle 24 by incorporating the predicted peak values from BMLD model.

prediction with the actual data. To avoid this, we initially make a lower bound prediction and an upper bound prediction for the forecast, only to take care of the shift in the length of the cycle due to normalization. For this, from the length of all the previous 23 sunspot cycle it can be computed that the average cycle length is 132 months with a standard deviation of 14 months. So we can compute an upper bound of sunspot cycle length as $132+14=146$ months and a lower bound as $132-14=118$ months. Using this we can have a coefficient of renormalization of 1.1061 for upper bound and 0.8939 for lower bound. Using these two we predict an envelope for the predicted cycle 24 and plotted the observed cycle 24 over this envelop. Then it is found that, cycle 24 is well close to the lower bound profile. Figure 6 gives the envelop (lower and upper bound) for cycle 24 .

It is seen that the predicted peak amplitude of cycle 24 is 78 . It may be seen that this prediction is carried out when only 24 months of data were available. The predicted peaks of cycle 24 along with their epoch of occurrence together with actual 24 cycle values are given in the table 6 . Figure 7 shows the upper bound and lower bound prediction of the entire sunspot cycle 24 along with the actual measured sunspot number values. Error in prediction is computed for this procedure. For cycle 24, the prediction error is plotted in figure 8 . It may be noted that, at the time of this study observed data up to May 2016 are used for computing the error. That is, only initial 90 points in cycle 24 are considered for the error plotting. Here, it can be seen

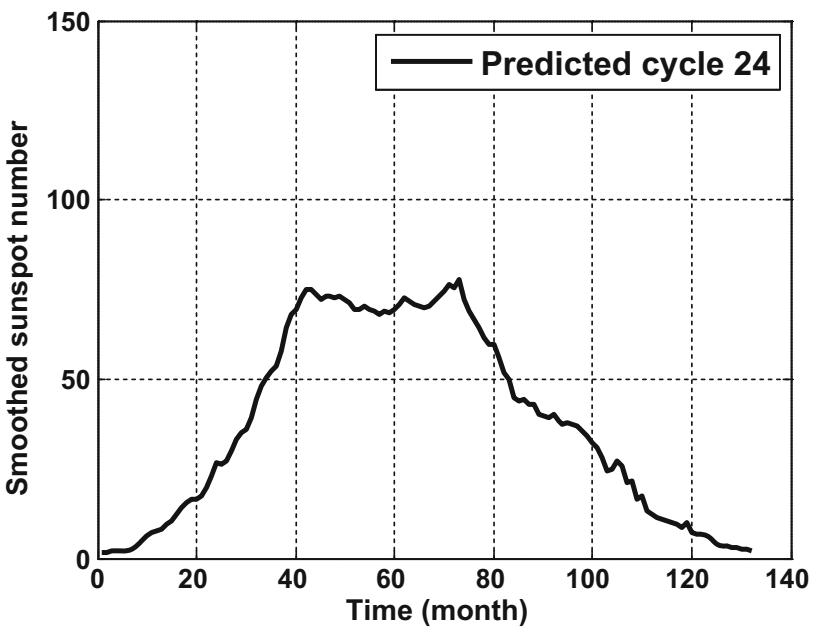

Figure 5. Improved prediction of the entire sunspot cycle 24.

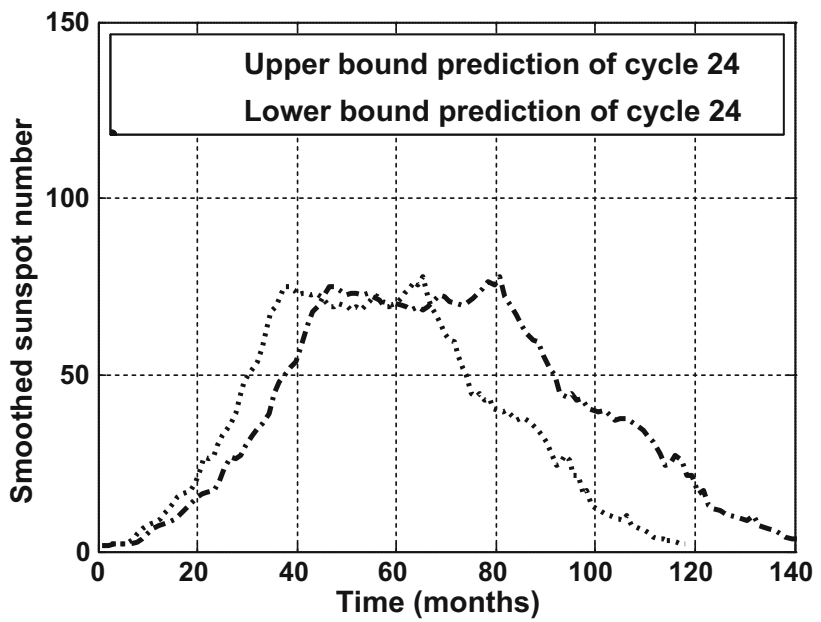

Figure 6. Upper bound and lower bound prediction of the entire sunspot cycle 24 .

that when more and more data get generated, the prediction error vanishes; even though the maximum prediction error is of \pm 25 in the peak solar activity regime. Figure 9 gives the histogram plot of the error computed for the prediction of cycle 24 throughout the cycle.

\section{Validation of the methodology - prediction of cycles 22 and 23}

In order to check the consistency of the present technique in predicting the sunspot cycle, this technique has been validated by predicting some of the past completed cycles. Using the proposed methodology, validation of the techniques has been done by predicting the past sunspot cycles 22 and 23 . The data matrix have been derived by considering the previous completed cycle data. Figure 10 shows 
Table 6. Predicted sunspot numbers at the two peaks for cycle 24.

\begin{tabular}{lclcl}
\hline Cycle 24 & $\begin{array}{c}\text { First peak } \\
\text { maximum }\end{array}$ & Epoch of first peak & $\begin{array}{c}\text { Second peak } \\
\text { maximum }\end{array}$ & Epoch of second peak \\
\hline Prediction & 72 & $\begin{array}{l}46 \text { months from the } \\
\text { cycle 24 beginning }\end{array}$ & 78 & $\begin{array}{l}74 \text { months from the cycle } \\
24 \text { beginning }\end{array}$ \\
Actual & 66 & $\begin{array}{l}39 \text { months from the } \\
\text { cycle 24 beginning }\end{array}$ & 80 & $\begin{array}{l}64 \text { months from the cycle } \\
24 \text { beginning }\end{array}$ \\
\hline
\end{tabular}

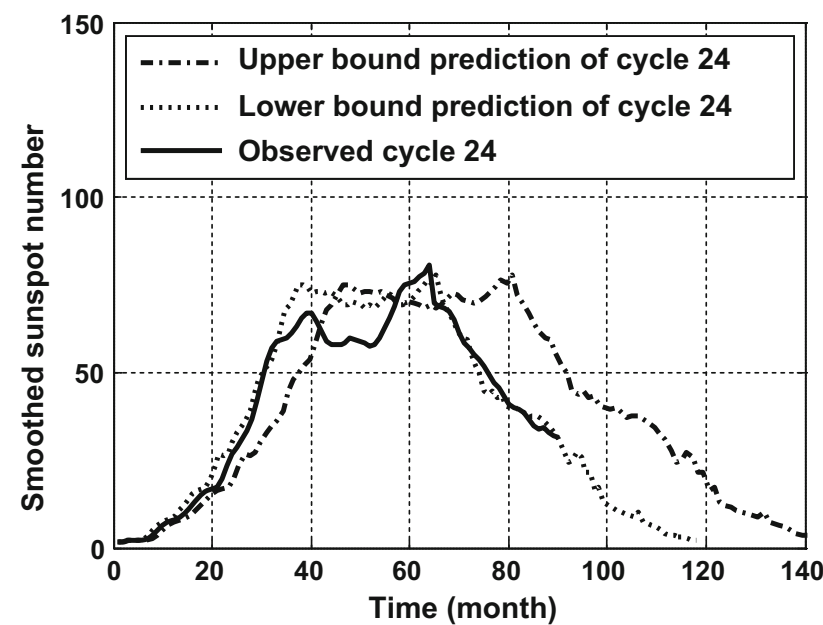

Figure 7. Upper bound and lower bound prediction of the entire sunspot cycle 24 along with the actual measured sunspot number values.

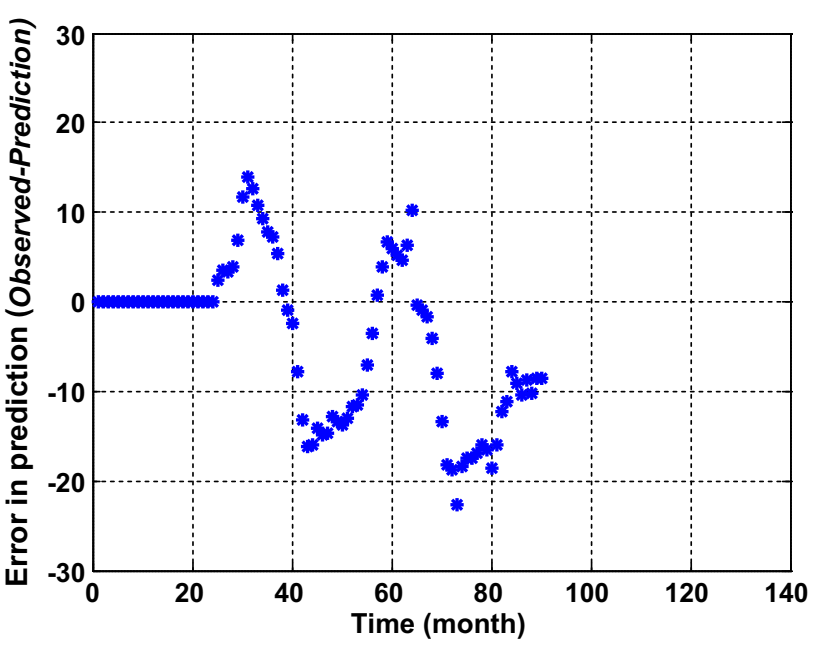

Figure 8. Prediction error of sunspot cycle 24 due to the final prediction at each month. Observed data considered up to May 2016 (i.e., only initial 90 points in cycle 24 are considered).

the prediction of sunspot cycle 23 and figure 11 shows error in prediction of sunspot numbers of cycle 23. Figure 12 shows the prediction of sunspot cycle 22 . For validating the cycles 22 and 23 , the previous cycles were used to generate the matrix,

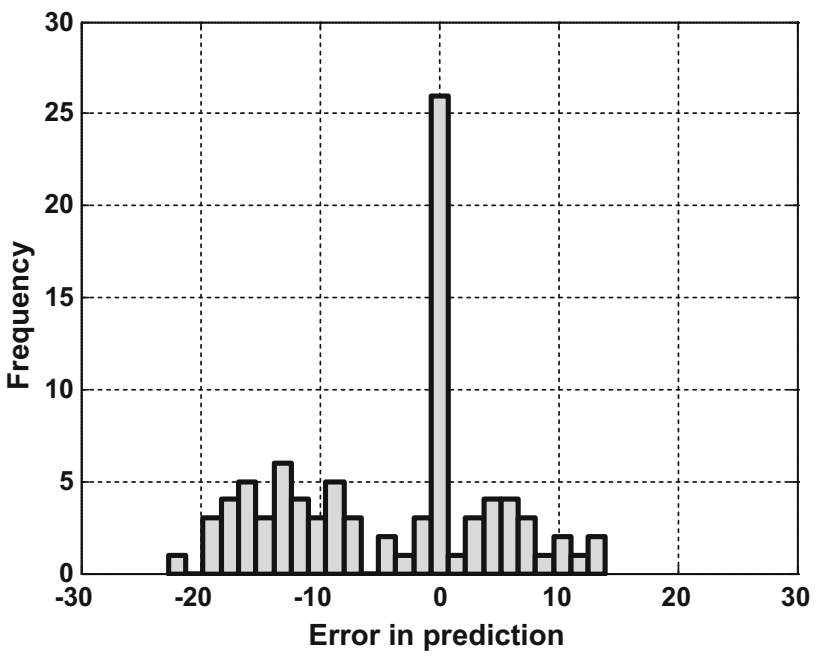

Figure 9. Histogram of prediction error of sunspot cycle 24 .

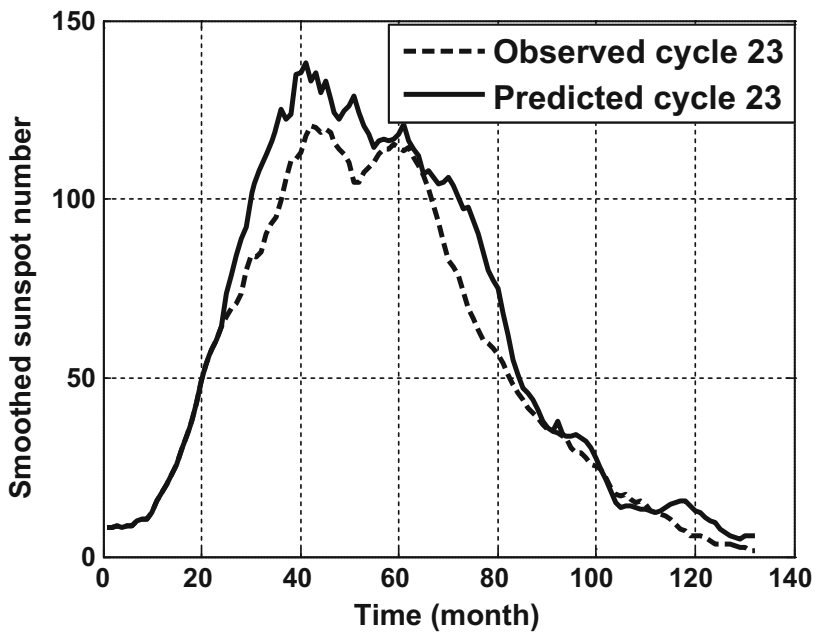

Figure 10. Prediction of the entire sunspot cycle 23 along with the actual measured sunspot number values.

and the initial 24 months of data from the cycle to be predicted is taken as the input for the algorithm. The predicted peak of the smoothed sunspot number cycle 23 is 138 , while the observed peak value is 120. The predicted peak of the smoothed sunspot number cycle 22 is 162 while the observed peak value is 156 . 


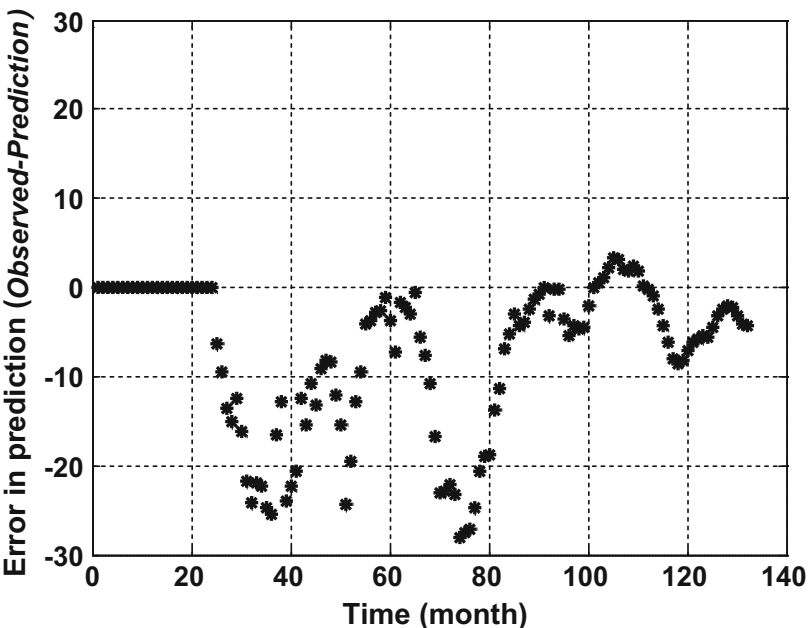

Figure 11. Prediction error of sunspot cycle 23 after the improved prediction at each month.

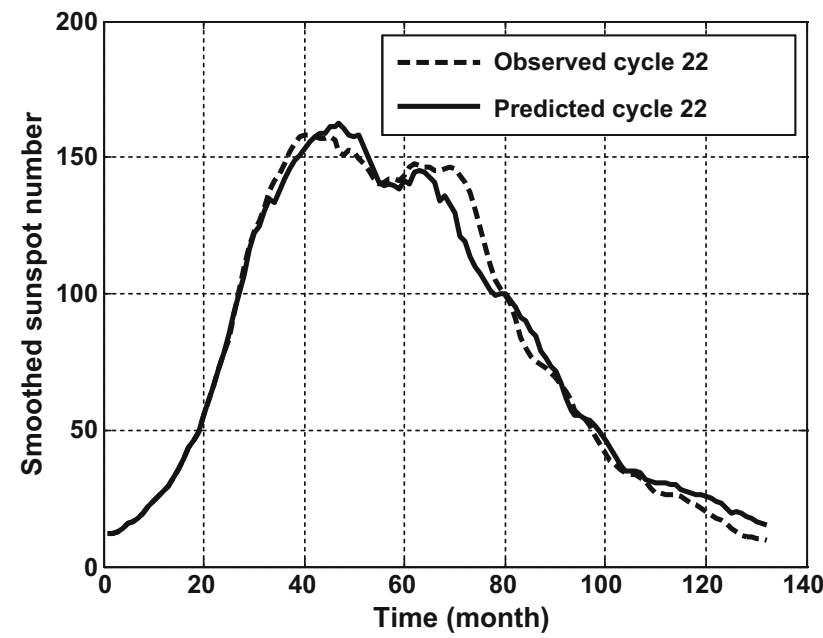

Figure 12. Prediction of the entire sunspot cycle 22 along with the actual measured sunspot number values.

\section{Prediction of cycle 25}

Using the same technique, an attempt has been made to predict the upcoming solar cycle 25 . The first step we need here is the initial 24 months of data. In fact, at present (in 2017) we are in the final descent phase (solar minimum) of cycle 24 . Hence, for making a prediction we need 24 months of sunspot number profile. Since we do not have the actual data, we can make a preliminary level estimate of the initial 24 months of data for cycle 25 by averaging the previous some selected cycles. Here, since it is found that for the slow rising and fast rising class different sunspot number data profile for initial 24 months, our averaging has been done separately for slow rising class and for fast rising class. In this way, the averaged initial profile are

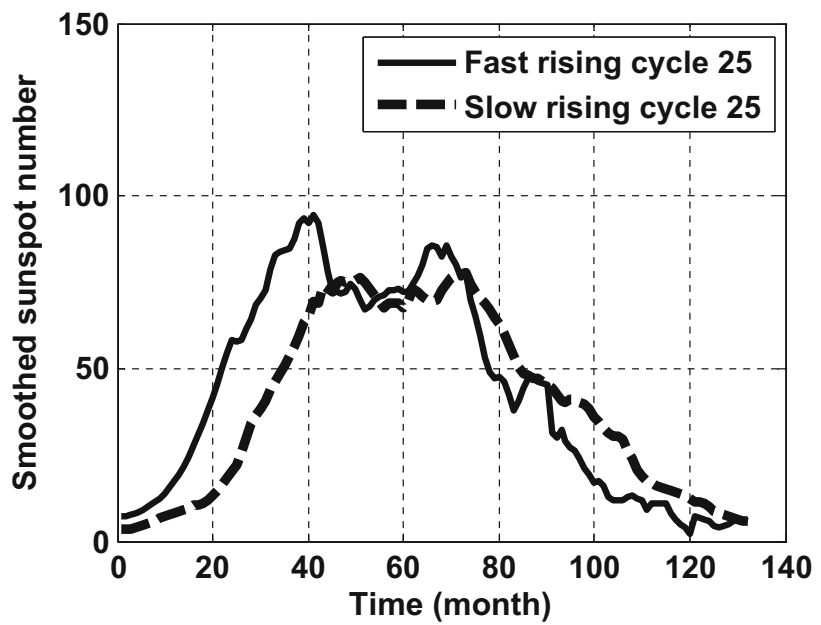

Figure 13. Prediction of the entire sunspot cycle 25 for slow rise and fast rise.

computed one as an upper bound corresponding fast rising class and a lower bound profile corresponding to slow rising class. Using these profiles, we made two predictions, one for a fast rise cycle 25 and another one for the slow rise cycle 25 . The prediction for cycle 25 is given in figure 13. According to our prediction, the sunspot cycle 25 also will be a small cycle with peak smoothed sunspot number of about 95 for a fast rising cycle. When cycle 25 data are available, the prediction can be performed and the prediction will lie within the band shown in figure 13.

\section{Conclusion}

A new prediction model for the sunspot number cycles based on multiple regression imputation technique and BMLD function has been proposed here. Unlike other predictions reported in the literature, in this study a novel prediction for cycle 24 have been presented. The full cycle 24 including the double peaks have been forecasted by using only the initial 24 months of cycle 24 . This technique predicts accurately the behaviour of sunspot cycle well ahead its maximum solar activity period. Moreover, unlike other predictions, using this technique we could predict the entire sunspot cycle as a complete profile which is very much useful in satellite orbital decay prediction and lifetime estimation process. In the multiple regression part, EM algorithm is utilized for predicting the future cycle. This initial prediction is modified again by assuming two peak values at 41st and 65th month. Again prediction of cycle 24 has been done using the multiple regression technique with these peak values. 
This is essentially for picking up the Gnevyshev gap phenomena. In this way, the prediction shows that cycle 24 may peak upto 78 in the second peak. Later it is found as true, the actual peak value of cycle 24 is 80 . The present study predict cycle 24 may have a double peak and the second peak may be stronger than the first one. This was predicted well by the BMLD model also. This methodology has been validated by predicting the past sunspot cycles 22 and 23 .

An attempt has also been made to predict the sunspot cycle 25. According to our prediction, the sunspot cycle 25 also will be a small cycle with a peak smoothed sunspot number in the range of 75 to 95 . By combining the multiple regression model and BMLD model we could predict the cycle 24 more accurately. These predicted sunspot cycle profile can be used for predicting the solar flux intensity, and it can be directly used in satellite orbital lifetime prediction tools.

\section{Acknowledgment}

The authors are thankful to the esteemed reviewers for their critical review and constructive comments to improve the paper.

\section{References}

Choudhuri A R, Chatterjee P and Jiang J 2007 Predicting solar cycle 24 with a solar dynamo model; Phys. Rev. Lett. 98131103.

Choudhuri A R 2014 The irregularities of the sunspot cycle and their theoretical modeling; Indian J. Phys. 88 877884.

Cliverd M A, Clarke E, Ulich T, Linthe J, Rishbeth H and Jarvis M J 2006 Predicting solar cycle 24 and beyond; Space Weather 4 S09005.

Dabas R S, Sharma K, Das R M, Pillai K G M, Chopra P and Sethi N K 2008 A prediction of solar cycle 24 using a modified precursor method; Sol. Phys. 250(1) 171-181.

de Mayer F 2003 A transfer function model for the sunspot cycle; Sol. Phys. 217 349-366.

Dikpati M, De Toma G and Gilman P A 2006 Predicting the strength of solar cycle 24 using a flux-transport dynamobased tool; Geophys. Res. Lett. 33(5) L05102.

Drecher P E, Little R P and Wittenstein G 1980 Skylab orbital lifetime prediction and decay analysis; NASA Technical Memorandum 78308, Alabama, Scientific and Technical Information Branch, NASA.

Duhau S 2003 An early prediction of maximum sunspot number in solar cycle 24; Sol. Phys. 213(1) 203-212.

Georgieva K and Kirov B 2011 Solar dynamo and geomagnetic activity; J. Atmos. Sol Terr. Phys. 73(2-3) $207-222$.
Gnevyshev M N 1967 On the 11-years cycle of solar activity; Sol. Phys. 1(1) 107-120.

Hathaway D H, Wilson R M and Reichmann E J 1994 The shape of the sunspot cycle; Sol. Phys. 151(1) 177-190.

Hathaway D H 2010 The solar cycle; Living Rev. Sol. Phys. 7 1, http://www.livingreviews.org//rsp-2010-1.

Hiremath K M 2006 The solar cycle as a forced and harmonic oscillator: Long term variation of the amplitudes, frequencies and phases; Astron. Astrophys. 452(2) 591-595.

Hiremath K M 2008 Prediction of solar cycle 24 and beyond; Astrophys. Space Sci. 314(1-3) 45-49.

Javaraiah J 2008 Predicting the amplitude of a solar cycle using north-south asymmetry in the previous cycle: II. An improved prediction for solar cycle 24; Sol. Phys. 252 419-439.

Joshi B, Bhattacharyya R, Pandey K K, Kushwaha U and Jae Moon Y 2015 Evolutionary aspects and north-south asymmetry of soft X-ray flare index during solar cycles 21, 22, and 23; Astron. Astrophys. 582 A4.

Kane R P 1999 Prediction of the sunspot maximum of solar cycle 23 by extrapolation of spectral components; Sol. Phys. 189 217-224.

Kane R P 2005 Which one is the Gnevyshev gap?; Sol. Phys. 229(2) 387-407.

Kane R P 2007 A preliminary estimate of the size of the coming solar cycle 24 based on Ohl's precursor method; Sol. Phys. 243(2) 205-217.

Kilicik A, Anderson C N K, Rozelot J P, Ye H, Sugihara G and Ozguc A 2009 Non-linear prediction of solar cycle 24; Astrophys. J. 693(2) 1173-1177.

King Hele D 1987 Satellite orbits in an atmosphere: Theory and applications; Blackie and Sons Ltd, Glasgow, UK.

Kitiashvili I N and Kosovichev A G 2008 Prediction of sunspot cycles by data assimilation method; Technical report, Stanford University, Stanford CA.

Little R J A and Rubin D B 1987 Statistical analysis with missing data; John Wiley \& Sons, New York.

Obridko V N and Shelting B D 2008 On prediction of the strength of the 11-year solar cycle; Sol. Phys. 248 191-202.

Osherovich V and Fainberg J 2008 New method of solar maximum prediction with application to the next solar cycle; Eos. Trans. AGU 89131505.

Parsapoor M, Bilstrup U and Svensson B 2015 Prediction of solar cycle 24; International Joint Conference on Neural Networks (IJCNN 1025), Killarney, Ireland, IEEE Press, $1-8$.

Pesnell W D 2008 Predictions of solar cycle 24; Sol. Phys. $252209-220$.

Petrovay K 2010 Solar Cycle Prediction; Living Rev. Sol. Phys. 7 6, http://www.livingreviews.org/lrsp-2010-6.

Qian L, Roble R G, Solomon S C and Kane T J 2006 Calculated and observed climate change in the thermosphere and a prediction for solar cycle 24; Geophys. Res. Lett. 33 L23705.

Quassim M S, Attia A F and Elminir H K 2007 Forecasting the peak amplitude of the 24 th and 25 th sunspot cycles and accompanying geomagnetic activity; Sol. Phys. 243 253-258.

Sabarinath A and Anilkumar A K 2008 Modeling of sunspot numbers by a modified binary mixture of Laplace distribution function; Sol. Phys. 250 183-197. 
Sabarinath A and Anilkumar A K 2011 A stochastic prediction model for the sunspot cycles; Sol. Phys. 273 $255-265$.

Saxena A K, Tiwari C M and Singh P R 2016 Variations in solar cycles 22, 23, \& 24 and their effect on Earth's climate; Int. J. Astron. Astrophys. 6 813.

Schatten K 2005 Fair space weather for solar cycle 24; Geophys. Res. Lett. 32(21) L21106.
Svalgaard L, Cliver E W and Kamide Y 2005 Sunspot cycle 24: Smallest cycle in 100 years?; Geophys. Res. Lett. 32(1) L01104.

Withbroe G L 1989 Solar activity cycle: History and predictions; J. Spacecr. Rockets 26(6) 394-402.

Wang J L, Gong J C, Liu S Q, Le G M and Sun J L 2002 The prediction of maximum amplitudes of solar cycles and the maximum amplitude of solar cycle 24; Chinese J. Astron. Ast. 2(6) 557-562.

Corresponding editor: Suresh BABU 